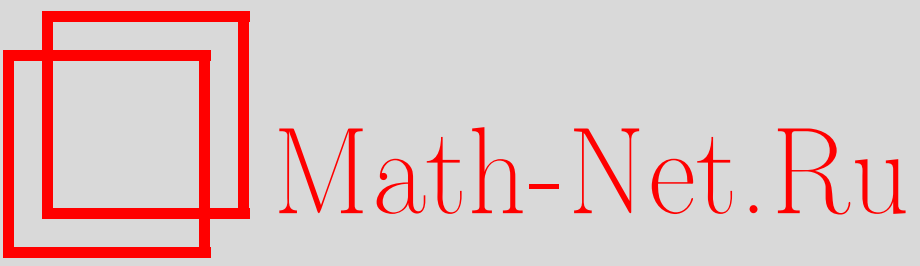

Г. Аманн, Нелокальные квазилинейные параболические уравнения, УМH, 2005, том 60 , выпуск $6,21-32$

DOI: https://doi.org/10.4213/rm1674

Использование Общероссийского математического портала Math-Net.Ru подразумевает, что вы прочитали и согласны с пользовательским соглашением

http://www.mathnet.ru/rus/agreement

Параметры загрузки:

IP: 3.89 .185 .249

26 апреля 2023 г., 11:50:28 


\title{
НЕЛОКАЛЬНЫЕ КВАЗИЛИНЕЙНЫЕ ПАРАБОЛИЧЕСКИЕ УРАВНЕНИЯ
}

Г. АМАнН

\begin{abstract}
Мы приводим обзор наиболее общих подходов к теории квазилинейных параболических эволюционных уравнений, обсуждаем их преимущества и недостатки и представляем совершенно новый подход, основанньй на максимальной $L_{p}$-регулярности. Наши общие резултаты применимы прежде всего к параболическим началњно-краевым задачам, нелокальным по времени. Это иллюстрируется указанием их важности для квазилинейных параболических уравнений с памятью и, в частности, для регуляризованных по времени вариантам уравнения Перона-Малика для обработки изображений.

Библиографиия: 42 названия.
\end{abstract}

\section{СОДЕРЖАНИЕ}

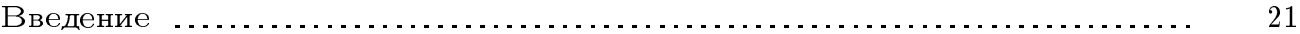

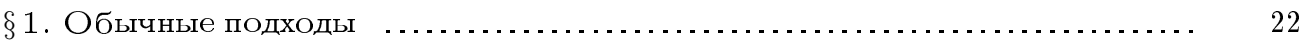

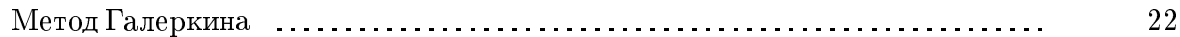

Монотонные операторы и аккретивные полугруппы …............... 23

Априорные оценки и техника продолжений Лере-Шаудера .............. 23

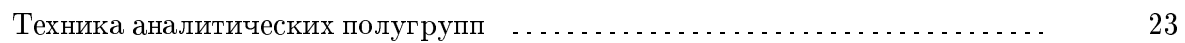

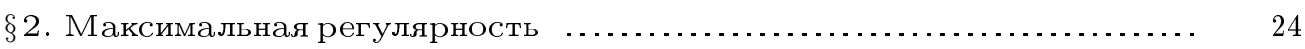

Максимальная непрерьвная регулярность …..................... 25

Максималная регулярность по Гёльдеру ............................. 25

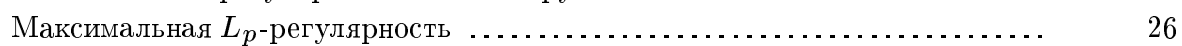

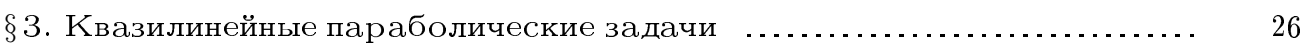

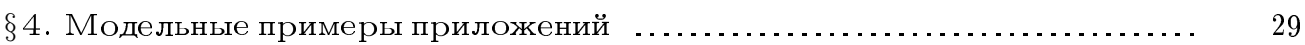

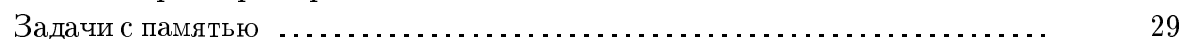

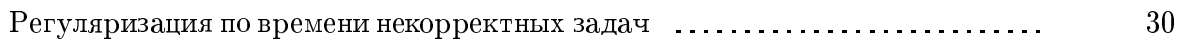

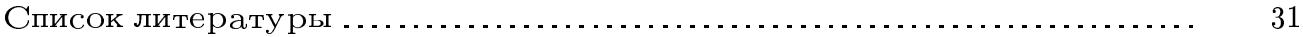

\section{Введение}

В этой статье мы обсуждаем новый подход к абстрактному квазилинейному параболическому уравнению

$$
\dot{u}+A(u) u=F(u) \text { на }(0, T), u(0)=u^{0},
$$


где $T$ - фиксированное положительное число. Формулировка (0.1) включает в себя богатое многообразие конкретных задач, в особенности параболические начально-краевые задачи вида

$$
\left.\begin{array}{c}
\partial_{t} u-\nabla \cdot(\mathbf{a}(u) \nabla u)=\mathbf{f}(u) \text { на } \Omega \times(0, T), \\
\chi \vec{\nu} \cdot \mathbf{a}(u) \nabla u+(1-\chi) u=\chi \mathbf{g}(u) \text { на } \Gamma \times(0, T), \\
u(\cdot, 0)=u^{0} \text { на } \Omega,
\end{array}\right\}
$$

где $\chi \in\{0,1\}, \Omega$ - ограниченная гладкая область с гранищей $\Gamma$ и $\vec{\nu}$ - внешняя нормаль к Г. В отличие от стандартных классических постановок, для нас особенно интересны ситуации, в которых $\mathbf{a}, \mathbf{f}$ и $\mathbf{g}-$ нелокальные функции от $u$. Конечно, матрица $\mathbf{a}(u)$ должна быть равномерно положительно определена на $\bar{\Omega}$ при любом допустимом выборе величины $u$. Следует также заметить, что (0.2) включает и системы, если использовать очевидные интерпретации.

В следуюшем параграфе мы приводим обзор известных стандартных подходов к задачам (0.1) и (0.2). В $§ 2$ мы кратко обсуждаем понятия максимальной регулярности. В $\S 3$ мы приводим нашу основную новую абстрактную теорему, а в последнем параграфе мы указываем некоторые приложения, иллюстрируюшие силу и новизну нашего подхода.

\section{§ 1. Обычные подходы}

Следующие довольно простые замечания относятся главным образом к $(0.2)$ в простом скалярном случае, когда $\mathbf{a}, \mathbf{f}$ и $\mathbf{g}$ - локальные функции.

По-видимому, наиболее известным подходом к (линейным) параболическим задачам является

Метод Галеркина. Насколько нам известно, для нелинейных эволюционных уравнений этот метод был впервые использован Э. Хопфом [24], который применил его для доказательства сушествования слабых решений уравнения Навье-Стокса. Он был затем широко популяризирован М.И. Вишиком и О.А. Ладыженской [40], О.А. Ладыженской [26] и рядом других авторов, в особенности Ж.-Л. Лионсом [30]. В контексте линейных задач Лионс развил этот подход до мощной абстрактной теории. С тех пор многие авторы распространяли этот метод на нелинейные задачи (см., например, [13], [42] и многочисленные исследовательские работы), где, в большинстве случаев, изучались полулинейные уравнения. (Здесь и в дальнейшем мы ограничиваемся указанием ссылок на легко доступные работы, главным образом на книги. Это не значит, что мы обеспечиваем корректное историческое изложение развития исследований. Мы предоставляем читателю проследить литературу до истоков конкретной теории.)

Метод Галеркина имеет важное преимушество:

- он относительно прост.

Однако он имеет ряд серьезных недостатков. А именно:

- он ограничен в основном постановками задач в гильбертовом пространстве, что сводится к слабой $H^{1}$-постановке в случае задачи $(0.2)$;

- он может быть применен только к коэрцитивньм задачам, и поэтому нельзя работать с обшими системами;

- в случае постановки задачи в гильбертовом пространстве нужно накладывать сильные ограничения на рост нелинейностей $f$ и $g$.

Другой хорошо известный абстрактньй метод имеет своим основанием 
Монотонные операторы и аккретивные полугруппы. В контексте эволюционных уравнений этот метод был разработан, в частности, $\Phi$. Бениленом [10], Х. Брезисом [11] и М. Кренделлом [16] и был уточнен и развит во многих работах. Хотя он приводит к сравнительно хорошим результатам, главным образом для вырожденных задач, его область применения жестко ограничена тем фактом, что он существенно основан на свойствах монотонности и принщипах максимума. Таким образом,

- этот метод нельзя применять ни к обшим системам, ни к задачам высших порядков,

если только не выполнены некоторые весьма специальные структурные условия.

Что касается стандартной (локальной) квазилинейной параболической задачи $(0.2)$, есть фундаментальная и глубокая работа, в основании которой лежат

Априорные оценки и техника продолжений Лере-Шаудера. Эти методы были развиты в начале второй половины прошлого столетия, главным образом О. А. Ладыженской, Н. Н. Уральцевой и В. А. Солонниковым, и изложены в их монументальной работе [27]. Недавние обобщения и уточнения, касаюшиеся в том числе некоторых вырожденных задач, приведены в книге Г. Либермана [29]. Результаты, полученные этим методом, оптимальны. Однако метод имеет следуюшие недостатки:

- по сушеству эта техника ограничивается скалярньм случаем и не может быть применена к общим системам,

- трудно исследовать задачи, в которых происходит взрьв.

Наиболее общим и гибким подходом к абстрактному параболическому эволюционному уравнению (0.1), применимьм, в частности, к его конкретной реализации (0.2), является метод, в основе которого лежит

Техника аналитических полугрупп. Исследования в этом направлении были независимо инициированы Т. Като [25] и П. Е. Соболевским [39]. Эти авторы рассматривали главным образом линейную теорию, являюшуюся основой изучения квазилинейных задач, и получили предварительные результаты для квазилинейных задач. Аналитические полугруппы играют решаюшую роль в геометрической теории почти линейных параболических задач Д. Хенри [22] . Удовлетворительная общая абстрактная теория квазилинейных параболических эволюционных уравнений была развита лишь сравнительно недавно автором [2], [3] и А. Лунарди [31], причем Лунарди ограничилась теорией в гёльдеровых пространствах.

Преимущества подхода, использующего технику аналитических полугруп, многообразны. В частности,

- эта техника применима как к слабым, так и к классическим постановкам квазилинейных параболических задач [2], [8],

- она применима к обшим (параболическим по Петровскому) системам [2], [28],

- она допускает геометрическую теорию квазилинейных параболических эволюционных уравнений в духе геометрической теории обыкновенных дифференциальных уравнений [1], [2], [37], [38],

- это - очень гибкая теория, применимая к широкому семейству нестандартных моделей, включая граничные задачи со свободными и подвижными границами [19], уравнения с динамическими граничньми условиями [18], сингулярные задачи Коши [20], уравнения с бесконечньм - даже несчетным - набором уравнений [4], и тому подобные задачи, решение которых недостижимо другими методами. 
В принципе, подход к задаче (0.1) с точки зрения техники аналитических полугруп очень прост и непосредствен. Он состоит из двух основных шагов, а именно,

- во-первых, нужно получить хорошую информацию о существовании, единственности и свойствах непрерывности для неавтономной линейной задачи

$$
\dot{u}+A(t) u=f(t) \text { на }(0, T), u(0)=u^{0} ;
$$

- во-вторых, используя первый шаг, нужно выделить подходяшие классы функщий $v$, которым может принадлежать предполагаемое решение (0.1); затем, обозначая через $u(v)$ единственное решение уравнения

$$
\dot{u}+A(v(t)) u=F(v(t)) \text { на }(0, T), \quad u(0)=u^{0},
$$

в этом классе, мы должны только найти неподвижную точку отображения $v \mapsto$ $u(v)$.

Конечно, приведенная выше схема чрезвычайно упрощена, и в ее реализации есть много трудностей, часть которых мы сейчас обсудим.

\section{§. Максимальная регулярность}

Полугрупповой подход к задаче (0.1), схема которого приведена вьше, использует следуюшие основные предположения:

(a) $E, E_{0}$ и $E_{1}$ - такие банаховы пространства, что $E_{1} \hookrightarrow E \hookrightarrow E_{0}$;

(б) $(x \mapsto A(x))$ - локально липшицево отображение из $E$ в $\mathscr{L}\left(E_{1}, E_{0}\right)$;

(в) для любого $x \in E$ оператор $-A(x)$ порождает сильно непрерьвную аналитическую полугруппу на $E_{0}$;

(г) $F$ - локально липшицево отображение из $E$ в $E_{0}$.

Как обычно, символ $\hookrightarrow$ означает непрерывное вложение, а $\mathscr{L}\left(E_{1}, E_{0}\right)$ - банахово пространство всех ограниченных линейных операторов из $E_{1}$ в $E_{0}$.

При этих условиях, а также в предположении, что $E$ - интерполяционное пространство между $E_{0}$ и $E_{1}$ (и при некоторых дополнительных незначительных технических уточнениях), можно показать, что задача (0.1) корректна и порождает локальньй полупоток на $E$ (cp. [2]). Хотя этот результат имеет многочисленные глубокие приложения, указанные вьше, тот факт, что $E$ должно быть промежуточным пространством между $E_{0}$ и $E_{1}-$ небольшое, но, в некоторых случаях, нежелательное ограничение. Интуитивно ясно, что оптимальная постановка возникает при $E=E_{1}$.

Схема, приведенная в начале предыдущего параграфа, показьвает, что глубокое понимание линейной задачи (1.1) является решаюшим для подхода в целом. Действительно, оказьвается, чтобы провести шаг 2 , т.е. чтобы гарантировать, что отображение неподвижной точки $v \mapsto u(v)$ корректно определено, нужно знать, что задача

$$
\dot{u}+A(t) u=f(t) \quad \text { на }(0, T), \quad u(0)=0,
$$

имеет единственное решение для каждой $f:(0, T) \rightarrow E$, если $f$ удовлетворяет подходяшим условиям регулярности по времени, и что решение принадлежит тому же классу регулярности. Точнее, положим $J:=[0, T)$ и предположим, что

$$
\begin{aligned}
& X_{j}(J) \text { - такие банаховы пространства, что } X_{j}(J) \hookrightarrow L_{1}\left(J, E_{j}\right), j=0,1 ; \\
& A \in L_{\infty}\left(J, \mathscr{L}\left(E_{1}, E_{0}\right)\right) \text { и }(u \mapsto A u) \in \mathscr{L}\left(X_{1}(J), X_{0}(J)\right),
\end{aligned}
$$


где, конечно, $(A u)(t):=A(t) u(t)$ для п.в. $t \in J$. Тогда говорят, что $A$ имеет максимальную регулярность относительно $\left(X_{1}(J), X_{0}(J)\right)$, если задача $(2.2)$ при каждом $f \in X_{0}(J)$ имеет единственное решение (в смысле обобщенных функций) $u \in$ $X_{1}(J)$, удовлетворяюшее условию $\dot{u} \in X_{0}(J)$. Заметим, что, в связи со второй частью (2.3), это означает, что все три члена в (2.2) имеют одну и ту же регулярность, т.е. $\dot{u}, A u, f \in X_{0}(J)$.

$\mathrm{K}$ сожалению, вообще говоря, максимальная регулярность не обязательно имеет место, даже если $A$ и $X_{j}(J)$ “довольно хорошие". Однако есть важные случаи, в которых это условие вьполняется. В дальнейшем мы их опишем.

Максимальная непрерывная регулярность. Конечно, наиболее естественньй выбор - это

$$
X_{j}(J):=C\left(J, E_{j}\right), \quad j=0,1 .
$$

В этом случае мы получаем классические решения

$$
u \in C\left(J, E_{1}\right) \cap C^{1}\left(J, E_{0}\right),
$$

если

$$
f \in C\left(J, E_{0}\right) .
$$

Очевидно, нужно предполагать, что отображение $t \mapsto A(t)$ тоже непрерьвно, а не только ограничено и измеримо. К сожалению, в этой простой и естественной постановке максимальная регулярность вьполняется только при сильных ограничениях на банахова пространства $E_{1}$ и $E_{0}$, при которых конкретные приложения оказываются затруднительными. В частности, максимальная регулярность никогда не вьполняется, если $E_{1} \neq E_{0}$ и $E_{0}$ реффлексивно. Таким образом, это исключает возможность рассматривать в теории дифференщиальных уравнений с частными производными столь важные пространства, как $L_{q}(\Omega)$ или $H_{q}^{-1}(\Omega)($ при $1<q<\infty)$. С другой стороны, (малые) пространства Никольского или пространства Гёльдера допустимы. Подробности см. в [3; $§$ III.3]. По поводу соответствующей нелинейной теории, относящейся к (0.1), мы отсылаем читателя к [15], где можно также найти ссылки на конкретные приложения.

Максимальная регулярность по Гёльдеру. Ограничения на основные банахова пространства $E_{0}$ и $E_{1}$ можно полностью опустить, если мы рассмотрим гёльдеровские решения, т.е. если мы положим

$$
X_{j}(J):=C^{\alpha}\left(J, E_{j}\right), \quad j=0,1,
$$

для некоторого $\alpha \in(0,1)$. (На самом деле нужно выбирать более сложные подпространства $C^{\alpha}\left(J, E_{j}\right)$, но мы не приводим точных деталей.) В этом случае можно развить удовлетворительную теорию даже для полностью нелинейного уравнения $\dot{u}=$ $\Phi(u)$, если предположить, что $\Phi \in C^{1}\left(E_{1}, E_{0}\right)$ и для любого $e \in E_{1}$ оператор $\Phi^{\prime}(e)$ порождает сильно непрерьвную аналитическую полугруппу на $E_{0}$. Этот подход был разработан, в частности, Г. Да Прато и А. Лунарди и изложен в [31]. Оказьвается, однако, что этот подход не очень хорошо приспособлен к квазилинейному случаю, где $\Phi(u)=-A(u) u+F(u)$. Кроме того, из-за высоких требований к регулярности нелегко применять этот метод к конкретным уравнениям или получать результаты о глобальном существовании, поскольку эта постановка требует априорных оценок в довольно сильных нормах. 
Максимальная $L_{p}$-регулярность. С современной точки зрения на теорию дифференциальных уравнений с частными производными, наиболее желательной является $L_{p}$-теория. Точнее, предположим, что $1<p<\infty$ и положим

$$
X_{j}(J):=L_{p}\left(J, E_{j}\right), \quad j=0,1 .
$$

Таким образом, мы теперь интересуемся $\mathscr{H}_{p}^{1}$-решениями, где

$$
\mathscr{H}_{p}^{1}:=\mathscr{H}_{p}^{1}\left(J,\left(E_{1}, E_{0}\right)\right):=L_{p}\left(J, E_{1}\right) \cap H_{p}^{1}\left(\stackrel{\circ}{J}, E_{0}\right)
$$

и $H_{p}^{1}\left(\stackrel{\circ}{J}, E_{0}\right)$ - соболевское пространство таких $E_{0}$-значных обобщенных функций (распределений) $u$ на $\stackrel{\circ}{J}$, что $u$ и $\dot{u}$ принадлежат $X_{0}(J)=L_{p}\left(J, E_{0}\right)$. В этом случае максимальная регулярность относительно $\left(X_{1}(J), X_{0}(J)\right)$ называется максимальной $L_{p}$-регулярностью на $J$ относительно $\left(E_{1}, E_{0}\right)$. Чтобы гарантировать вьполнение условия максимальной $L_{p}$-регулярности, нужно наложить некоторые условия на $A$, как и на соответствующие банаховы пространства $E_{1}$ и $E_{0}$. В автономном случае полная характеризация, принадлежащая Л. Вайсу [41], основана на понятии $R$-ограниченности, на новой теореме типа Михлина о мультипликаторах Фурье для операторнозначных символов и на некоторых результатах теории банаховых пространств. Мы здесь не будем вдаваться в детали и вместо этого сошлемся на подробное изложение [17]. Может быть, достаточно сказать - несколько неформально - что

максимальная $L_{p}$-регулярность вьполняется в “хороших" пространствах вроде $L_{q}(\Omega)$ и $H_{q}^{-1}(\Omega)$ и для 'хороших' операторов, похожих на операторы, индуцируемые в таких пространствах эллиптическими дифференциальными операторами.

Наконец, следует отметить, что во всех обсуждавшихся вьше случаях максимальной регулярности необходимо, чтобы оператор $-A(t)$ порождал аналитическую полугрупу на $E_{0}$ при любом $t \in J$.

\section{§. Квазилинейные параболические задачи}

Вернемся теперь к задаче (0.1) и обсудим ее корректность в рамках максимальной $L_{p}$-регулярности. Таким образом, мы предполагаем, что

$$
E_{0} \text { и } E_{1}-\text { такие банаховы пространства, что } E_{1} \stackrel{d}{\hookrightarrow} E_{0},
$$

где символ $\stackrel{d}{\hookrightarrow}$ означает плотное вложение. При фиксированной паре $\left(E_{1}, E_{0}\right)$ мы просто пишем

$$
\mathscr{H}_{p}^{1}(J):=\mathscr{H}_{p}^{1}\left(J,\left(E_{1}, E_{0}\right)\right)
$$

Положим также

$$
E:=\left(E_{0}, E_{1}\right)_{1 / p^{\prime}, p},
$$

где $(\cdot, \cdot)_{\theta, p}$ - функтор вещественной интерполящии с показателем $\theta \in(0,1)$ и параметром $p$. Таким образом, $E$ - следовое пространство для $\mathscr{H}_{p}^{1}(J)$, и

$$
E_{1} \stackrel{d}{\hookrightarrow} E \stackrel{d}{\hookrightarrow} E_{0}
$$

Как известно,

$$
\mathscr{H}_{p}^{1}(J) \hookrightarrow C(\bar{J}, E)
$$


(см., например, [3; теорема III.4.10.2]).

Благодаря (3.1) можно (и не очень трудно) воспользоваться полугрупповым подходом, основанным на предположении (2.1), при дополнительном допушении, что

$A(x)$ обладает максимальной $L_{p}$-регулярностью для любого $x \in E$.

Действительно, это было осушествлено $\Phi$. Клеманом и Ш. Ли [14] в конкретной постановке задачи и Я. Прюссом [36] в рамках абстрактной задачи. Доказательства в этой ограниченной постановке гораздо проще, чем аналогичные доказательства в общем случае [2], не накладывающем условия максимальной регулярности. Однако улучшения для приложений к конкретным квазилинейным параболическим задачам несушественны.

Таким образом, возникает следующий вопрос: в чем состоит оптимальная постановка теории максимальной $L_{p}$-регулярности для задачи $(0.1)$ ?

Очевидно, при поиске решений в $\mathscr{H}_{p}^{1}(J)$, минимальное требование имеет следующий вид:

$$
A \text { и } F \text { определены на } \mathscr{H}_{p}^{1}(J) \text { и } u^{0} \text { принадлежит } E \text {. }
$$

Это предположение существенно отличается от стандартных условий (2.1), где $A$ и $F$ определены на банаховом пространстве $E$ и, для заданной функции $u: J \rightarrow E$, отображение

$$
(A(u), F(u)): J \rightarrow \mathscr{L}\left(E_{1}, E_{0}\right) \times E_{0}
$$

определяется формулой

$$
(A(u), F(u))(t):=(A(u(t)), F(u(t))), \quad t \in J .
$$

Иными словами, $A$ и $F$ - локальные операторы (относительно $t$ ) в стандартной ситуации, тогда как $A$ и $F$ могут быть нелокальнымми отображениями в случае, если вьполняется (3.2).

Так как мы рассматриваем эволюционные уравнения, то в дополнение к (3.2) нам нужны дальнейшие условия, гарантирующие, что в любой момент времени не используется никакая информация о будушем. Таким образом, мы требуем, чтобы нелокальные отображения имели свойство Вольтерра:

для любого $S \in(0, T)$ и $u \in \mathscr{H}_{p}^{1}(J)$ имеем $(A(u), F(u)) \mid J_{S}=\left(A\left(u \mid J_{S}\right), F\left(u \mid J_{S}\right)\right)$.

Иными словами, ограничение функции $(A(u), F(u))$ на любой подынтервал в $J_{S}$ зависит только от ограничения $u$ на тот же самый интервал.

После этой подготовки мы можем сформулировать следующий результат о существовании, единственности и непрерывности.

Теорема 3.1. Пусть $\mathbf{J}:=J_{\mathbf{T}}:=[0, \mathbf{T})$ для некоторого $\mathbf{T} \in(0, \infty)$, причем $p \in$ $(1, \infty)$, а $E_{0}$ и $E_{1}-$ такие банахова пространства, что $E_{1} \stackrel{d}{\hookrightarrow} E_{0}$. Предположим также, что

(i) A - отображение Вольтерра из $\mathscr{H}_{p}^{1}(\mathbf{J})$ в $L_{\infty}\left(J, \mathscr{L}\left(E_{1}, E_{0}\right)\right)$, которое равномерно удовлетворяет условию Липшица на ограниченных множествax,

(ii) для любого $u \in \mathscr{H}_{p}^{1}(\mathbf{J})$ и любого $T \in \mathbf{J}$ линейное отображение $A(u) \mid J_{T}$ допускает максимальную $L_{p}$-регулярность на $J_{T}$ относительно $\left(E_{1}, E_{0}\right)$,

(iii) $F$ - отображсение Вольтерра из $\mathscr{H}_{p}^{1}(\mathbf{J})$ в $L_{p}\left(\mathbf{J}, E_{0}\right)$, и существует такое $r \in(p, \infty]$, что $F-F(0)$ удовлетворяет условию Липшица на ограниченных подмножествах в $\mathscr{H}_{p}^{1}(\mathbf{J})$ (как функиия со значениями в $\left.L_{r}\left(\mathbf{J}, E_{0}\right)\right)$,

(iv) $u^{0} \in\left(E_{0}, E_{1}\right)_{1 / p^{\prime}, p}$. 
Тогда справедливы следующие утверждения.

(1) Существует максимальное число $T^{*} \in(0, \mathbf{T}]$ и единственное решение $u^{*}$ задачи (0.1) на $J_{T^{*}}$, удовлетворяющее условию ${ }^{*} \in \mathscr{H}_{p}^{1}\left(J_{T}\right)$ при $0<T<T^{*}$, максимальное $\mathscr{H}_{p}^{1}$-решение.

(2) Если $T^{*}<\mathbf{T}$, mо $u^{*} \notin \mathscr{H}_{p}^{1}\left(J_{T^{*}}\right)$.

(3) Предположим, что $\left(\left(A_{j}, F_{j}, u_{j}^{0}\right)\right)$ - последовательность, удовлетворяющая условиям (i)-(iv) и сходящаяся $\kappa\left(A, F, u^{0}\right)$. Пусть $u_{j}^{*}-$ максимальное $\mathscr{H}_{p}^{1}-$ решение на $\left(0, T_{j}^{*}\right)$ задачи

$$
\dot{u}+A_{j}(u) u=F_{j}(u) \quad \text { на }(0, \mathbf{T}), \quad u(0)=u_{j}^{0} .
$$

Если $u^{*} \in \mathscr{H}_{p}^{1}(\mathbf{J})$, положим $S:=\mathbf{T} . \quad B$ противном случае вьбберем любое $S \in\left(0, T^{*}\right)$. Тогда существует такое $N \in \mathbb{N}$, ито $T_{j}^{*}>S$ при $j>N$, и последовательность $\left(u_{j}^{*}\right)$ сходится в $\mathscr{H}_{p}^{1}\left(J_{S}\right) \kappa u^{*}$.

ЗАмЕЧАНИЕ 3.2. (а) Чтобы придать точньй смысл сходимости последовательности данных $\left(\left(A_{j}, F_{j}, u_{j}^{0}\right)\right)$, положим

$$
[g]_{X, \mathscr{B}}:=\sup _{\substack{v, w \in \mathscr{B} \\ v \neq w}} \frac{\|g(v)-g(w)\|_{X}}{\|v-w\|_{\mathscr{H}_{p}^{1}(\mathbf{J})}},
$$

где $\mathscr{B}$ - ограниченное подмножество пространства $\mathscr{H}_{p}^{1}(\mathbf{J})$ и $X:=L_{\infty}\left(\mathbf{J},\left(E_{1}, E_{0}\right)\right)$ или $X:=L_{r}\left(\mathbf{J}, E_{0}\right)$. Тогда соотношение $\left(\left(A_{j}, F_{j}, u_{j}^{0}\right)\right) \rightarrow\left(A, F, u^{0}\right)$ означает, что $A_{j}(0) \rightarrow A(0)$ в $L_{\infty}\left(\mathbf{J}, \mathscr{L}\left(E_{1}, E_{0}\right)\right)$, а соотношение $\left[A_{j}-A\right]_{L_{\infty}\left(\mathbf{J}, \mathscr{L}\left(E_{1}, E_{0}\right)\right), \mathscr{B}} \rightarrow 0$ означает, что $F_{j}(0) \rightarrow F(0)$ в $L_{p}\left(\mathbf{J}, E_{0}\right)$ и

$$
\left[F_{j}-F\right]_{L_{r}\left(\mathbf{J}, E_{0}\right), \mathscr{B}} \rightarrow 0
$$

для любого ограниченного подмножества $\mathscr{B}$ пространства $\mathscr{H}_{p}^{1}(\mathbf{J})$, а также $u_{j}^{0} \rightarrow u^{0}$ в $\left(E_{0}, E_{1}\right)_{1 / p^{\prime}, p}$.

(б) Доказательство теоремы 3.1 приведено в [6] (см. [6; теоремы 2.1 и 3.1]). В этой статье предполагалось только то, что $A(u)$ удовлетворяет условию максимальной $L_{p^{-}}$ регулярности на $\mathbf{J}$ относительно $\left(E_{1}, E_{0}\right)$ для любого $u \in \mathscr{H}_{p}^{1}(\mathbf{J})$. Предположение, что это условие вьполняется для любого подынтервала $J_{T}$ в $\mathbf{J}$, отсутствует в [6]. Однако это условие требуется, поскольку доказательства в [6] используют лемму 4.1 в [5], которой недостаточно. Действительно, для любого $B \in L_{\infty}\left(\mathbf{J}, \mathscr{L}\left(E_{1}, E_{0}\right)\right)$, обладающего максимальной $L_{p}$-регулярностью на $\mathbf{J}$, доказательство этой леммы показывает, что линейная задача

$$
\dot{u}+B u=f \text { на }(0, T), \quad u(0)=0,
$$

имеет решение $u \in \mathscr{H}_{p}^{1}\left(J_{T}\right)$ для любого $T \in(0, \mathbf{T})$ и любого $f \in L_{p}\left(J_{T}, E_{0}\right)$, но вопрос о единственности остается открытым. (Впрочем, важность леммы 4.1 в [5] состоит в приведенных там равномерных оценках.)

(в) Следует отметить, что предположение (ii) можно ослабить следуюшим обра30M:

$(\mathrm{ii})^{\prime} A(u)$ имеет максимальную $L_{p}$-регулярность на $\mathbf{J}$ относительно $\left(E_{1}, E_{0}\right)$ для любого $u \in \mathscr{H}_{p}^{1}(\mathbf{J})$, и нуль является единственным решением в $\mathscr{H}_{p}^{1}\left(J_{T}\right)$ задачи

$$
\dot{v}+A(u) v=0 \text { на }(0, T), \quad v(0)=0,
$$

для любого $T \in(0, \mathbf{T})$. 
ДОКАЗАТЕЛЬСТВо ТЕОРЕМЫ 3.1. Очевидно, что из предположения (iі) следует (ii) ${ }^{\prime}$. Обратно, из (ii) ${ }^{\prime}$ и (доказательства) леммы 4.1 в [5] следует, что для любого $T \in(0, \mathbf{T})$ и $f \in L_{p}\left(J_{T}, E_{0}\right)$ линейная задача

$$
\dot{v}+A(u) v=f \text { на }(0, T), \quad v(0)=0,
$$

имеет решение $v \in \mathscr{H}_{p}^{1}\left(J_{T}\right)$.

\section{§4. Модельные примеры приложений}

Теорема 3.1 имеет многочисленные приложения к самым разным задачам. Для простоты мы ограничимся здесь краткими указаниями на некоторые задачи этого рода, относяшиеся к параболическим задачам, нелокальным во времени.

Задачи с памятью. Рассмотрим сначала уравнения типа реакции-диффузии вида

$$
\begin{gathered}
\partial_{t}(e(u))+\operatorname{div} \vec{\jmath}(u)=f(u) \text { на } \Omega \times(0, \infty), \\
\chi \vec{\nu} \cdot \vec{\jmath}(u)+(1-\chi) u=\chi g(u) \text { на } \Gamma \times(0, \infty), \\
u(\cdot, 0)=u^{0} \text { на } \Omega, \\
u=\bar{u} \text { на } \Omega \times(-\infty, 0) .
\end{gathered}
$$

Предположим, что

$$
\alpha, \beta \in L_{s, \mathrm{loc}}\left(\mathbb{R}^{+}\right) \text {для некоторого } s>1
$$

и

$$
a \in C^{2-}(\mathbb{R},(0, \infty))
$$

Как обычно, $C^{(k+1)-}$ - пространство всех $C^{k}$-функций, $k$-е производные которых локально липшицевы. Мы также предположим, что

$$
e(u)(\cdot, t):=u+\int_{-\infty}^{t} \alpha(t-\tau) u(\tau) d \tau=: u+\alpha *_{t} u
$$

и либо

$$
\vec{\jmath}(u):=-a\left(\beta *_{t} u\right) \nabla u
$$

либо

$$
\vec{\jmath}(u):=-a(u) \nabla u-\beta *_{t}(b(u) \nabla u)
$$

либо

$$
\vec{\jmath}(u):=-a(u) \nabla u-b(u(t-\tau)) \nabla u(t-\tau),
$$

где $\tau>0$ - фиксированное число и $b \in C^{2-}(\mathbb{R})$. Тогда $(4.1)$ - квазилинейная параболическая начально-краевая задача с запаздьванием. Системы такого типа возникают в различных приложениях, например, в теории теплопроводности в твердых телах с памятью. В этом случае $e$ - начальная энергия, $\vec{\jmath}$ - вектор теплового потока, а $u-$ температура (см., например, [21], [32], [34]). Другие примеры задач (4.1) возникают, например, в математической теории моделей климата (ср. [23]), математической биологии и теории управления. В другой публикации мы покажем, как можно применить теорему 3.1 к задаче (4.1), что приводит к значительным улучшениям и обобщениям известных результатов, а также открывает пути к исследованию задач, которые до сих пор были недосягаемы. 
Регуляризация по времени некорректных задач. Предположим, что

$$
a \in C^{2-}\left(\mathbb{R}^{+},(0, \infty)\right), \quad f \in C^{1-}\left(\bar{\Omega} \times \mathbb{R} \times \mathbb{R}^{n}\right),
$$

и рассмотрим модельную задачу

$$
\begin{gathered}
\partial_{t} u-\nabla \cdot\left(a\left(|\nabla u|^{2}\right) \nabla u\right)=f(x, u, \nabla u) \text { на } \Omega \times(0, \infty), \\
\chi \partial_{\vec{\nu}} u+(1-\chi) u=0 \text { на } \Gamma \times(0, \infty), \\
u(\cdot, 0)=u^{0} \text { на } \Omega .
\end{gathered}
$$

Предположим, что $S:=S(t)-$ регулярное множество уровня достаточно гладкого решения $u$ в некоторый момент времени $t \geqslant 0$. Тогда вблизи $S$ имеем

$$
\begin{aligned}
\nabla \cdot\left(a\left(|\nabla u|^{2}\right) \nabla u\right) & =a\left(|\nabla u|^{2}\right) \Delta u+2 a^{\prime}\left(|\nabla u|^{2}\right) D^{2} u \nabla u \cdot \nabla u \\
& =a\left(|\nabla u|^{2}\right) \Delta_{S} u+b\left(|\nabla u|^{2}\right) \partial_{\xi}^{2} u,
\end{aligned}
$$

где $D^{2} u$ - гессиан функции $u, \Delta_{S}$ - оператор Лапласа-Бельтрами поверхности $S$, векторное поле $\xi:=-\nabla u /|\nabla u|$ дает направление наискорейшего спуска для $u$ и

$$
b(s):=a(s)+2 s a^{\prime}(s), \quad s \geqslant 0 .
$$

Таким образом, может случиться, что $b(s)<0$ для точек $s$, принадлежаших некоторому интервалу $I$ на $(0, \infty)$. Следовательно, если $|\nabla u(x)| \in I$, то (4.4) есть обратная параболическая задача в направлении, перпендикулярном к $S$. Это так, в частности, при

$$
a(s):=1 /(1+s), \quad s \geqslant 0,
$$

где $b(s)<0$ при $s>1$. При таком выборе $a$, при $f=0$ и при граничных условиях Неймана задача (4.4) есть уравнение Перона-Малика [35], хорошо известное в обработке изображений и даюшее удивительно хорошие численные результаты. Однако развитой математической теории этого уравнения, обеспечивающей теоретическое оправдание этих результатов, нет. По этой причине предлагались различные модификации этого уравнения, в особенности метод пространственной регуляризации $\Phi$. Катте, П.-Л. Лионса, Ж.-М. Мореля и Т. Колла [12]. Эти авторы - а затем и практически все, кто занимался модификациями исходной модели Перона-Малика - заменяли $|\nabla u|^{2}$ в аргументе $a$ величиной $\left|\nabla u_{\sigma}\right|^{2}$, где $u_{\sigma}$ обозначает свертку по пространственному переменному с гауссианом с дисперсией $\sigma>0$. В этом случае нетрудно видеть, что задача (4.4) заменяется корректной задачей, близкой к уравнению П. Перона и Дж. Малика. К сожалению, пространственная реализация приводит к сглаживанию острых углов, и это приводит к нежелательному размьванию границ на изображениях (см. [7] по поводу числовых иллюстраций и более детального обсуждения).

В [7] мы предложили метод регуляризации по времени для уравнения Перона-Малика и родственных некорректных задач, обосновьваюший, в частности, модель, введенную М. Нитцбергом и Т. Шиота [33] (см. [9] по поводу теории последней системы в гёльдеровых пространствах). Наша модель не сглаживает острых углов, довольно гибкая и дает исключительно хорошие численные результаты. Ее математическим обоснованием является следствие приведенной ниже теоремы, связанной с вариантом задачи (4.4) с временнь́мм запаздьванием:

$$
\begin{gathered}
\partial_{t} u-\nabla \cdot\left(a\left(\theta *_{t}|\nabla u|^{2}\right) \nabla u\right)=f(x, u, \nabla u) \text { на } \Omega \times(0, \infty), \\
\chi \partial_{\vec{\nu}} u+(1-\chi) u=0 \text { на } \Gamma \times(0, \infty), \\
u=u^{0} \text { на } \Omega \times(-S, 0],
\end{gathered}
$$

где

$$
\theta \in L_{s}((0, S)) \text { для некоторых } s \in(1, \infty] \text { и } S \in(0, \infty) \text {. }
$$


Теорема 4.1. Предположим, что $p, q \in(1, \infty)$ удовлетворяет условию $2 / p+n / q<1$ и что выполняются условия (4.3) и (4.7). Тогда для любого $u^{0} \in H_{q}^{2}$, удовлетворяющего граничным условиям, существует максимальное число $T^{*} \in(0, \infty]$, для которого задача (4.6) имеет единственное решение $u^{*}$, принадлежащее пространству

$$
\mathscr{H}_{q, p}^{2,1}(\Omega \times(0, T)):=L_{p}\left((0, T), H_{q}^{2}\right) \cap H_{p}^{1}\left((0, T), L_{q}\right)
$$

для любого $T \in\left(0, T^{*}\right)$. Если $T^{*}<\infty$, то $u^{*} \notin \mathscr{H}_{q, p}^{2,1}\left(\Omega \times\left(0, T^{*}\right)\right)$. Если $f$ сублинейна по и и $\nabla$ и и если $\operatorname{supp}(\theta) \subset(\sigma, S)$ для некоторого $\sigma \in(0, S)$, то $u^{*}$ существует глобально, т.е. $T^{*}=\infty$.

ДокАЗАТЕльство. Первые два утверждения следуют из более общей теоремы 4.1 в [7], доказательство которой основано на теореме 3.1. По поводу последнего утверждения мы отсылаем читателя к замечанию 5.1(b) в [7].

\section{СПИСОК ЛИТЕРАТУРЫ}

[1] H. Amann. Hopf bifurcation in quasilinear reaction-diffusion systems // Lecture Notes in Math. 1991. V. 1475. P. 53-63.

[2] H. Amann. Nonhomogeneous linear and quasilinear elliptic and parabolic boundary value problems // Function Spaces, Differential Operators and Nonlinear Analysis (Friedrichroda, 1992). Stuttgart: Teubner, 1993. P. 9-126. (Teubner-Texte Math. V. 133.)

[3] H. Amann. Linear and Quasilinear Parabolic Problems. V. I: Abstract Linear Theory. Boston: Birkhäuser, 1995. (Monogr. Math. V. 89.)

[4] H. Amann. Coagulation-fragmentation processes // Arch. Ration. Mech. Anal. 2000. V. 151. № 4. P. 339-366.

[5] H. Amann. Maximal regularity for nonautonomous evolution equations // Adv. Nonlinear Stud. 2004. V. 4. № 4. P. 417-430.

[6] H. Amann. Quasilinear parabolic problems via maximal regularity // Adv. Differential Equations. 2005. V. 10. № 10. P. 1081-1110.

[7] H. Amann. Time-delayed Perona-Malik type problems // Preprint, http://www.math. unizh.ch/amann/publications.html.

[8] H. Amann, P. Quittner. Semilinear parabolic equations involving measures and low regularity data // Trans. Amer. Math. Soc. 2004. V. 356. № 3. P. 1045-1119.

[9] A. Belahmidi. Équations aux dérivées partielles appliquées à la restoration et à l'agrandissement des images // PhD thesis. Paris: CEREMADE, Université Paris-Dauphin, 2003; http://tel.ccsd.cnrs.fr.

[10] Ph. Bénilan. Équations d'évolution dans un espace de Banach quelconque et applications // PhD thesis. Orsay: Univ. Paris, 1972.

[11] H. Brézis. Opérateurs maximaux monotones et semi-groupes de contractions dans les espaces de Hilbert. Amsterdam: North-Holland, 1973.

[12] F. Catté, P.-L. Lions, J.-M. Morel, T. Coll. Image selective smoothing and edge detection by nonlinear diffusion // SIAM J. Numer. Anal. 1992. V. 29. № 1. P. 182-193.

[13] M. Chipot. Elements of Nonlinear Analysis. Basel: Birkhäuser, 2000.

[14] Ph. Clément, Sh. Li. Abstract parabolic quasilinear equations and application to a groundwater flow problem // Adv. Math. Sci. Appl. 1993/94. V. 3. Special Issue. P. 17-32.

[15] Ph. Clément, G. Simonett. Maximal regularity in continuous interpolation spaces and quasilinear parabolic equations // J. Evol. Equ. 2001. V. 1. № 1. P. 39-67.

[16] M. G. Crandall. Nonlinear semigroups and evolution governed by accretive operators // Nonlinear Functional Analysis and Its Applications, Part 1 (Berkeley, Calif., 1983). Providence, RI: Amer. Math. Soc., 1986. P. 305-337. (Proc. Sympos. Pure Math. V. 45.)

[17] R. Denk, M. Hieber, J. Prüss. $\mathscr{R}$-boundedness, Fourier multipliers and problems of elliptic and parabolic type // Mem. Amer. Math. Soc. 2003. V. 166. № 788.

[18] J. Escher. Quasilinear parabolic systems with dynamical boundary conditions // Comm. Partial Differential Equations. 1993. V. 18. № 7-8. P. 1309-1364. 
[19] J. Escher, G. Simonett. Moving surfaces and abstract parabolic evolution equations // Topics in Nonlinear Analysis. Basel: Birkhäuser, 1999. P. 183-212. (Progr. Nonlinear Differential Equations Appl. V. 35.)

[20] P. Guidotti. Singular quasilinear abstract Cauchy problems // Nonlinear Anal. 1998. V. 32. № 5. P. 667-695.

[21] M.E. Gurtin, A. C. Pipkin. A general theory of heat conduction with finite wave speeds // Arch. Ration. Mech. Anal. 1968. V. 31. P. 113-126.

[22] Д. Хенри. Геометрическая теория полулинейных параболических уравнений. М.: Мир, 1985.

[23] G. Hetzer. Global existence, uniqueness, and continuous dependence for a reaction-diffusion equation with memory // Electron. J. Differential Equations. 1996. № 05 (electronic).

[24] E. Hopf. Über die Anfangswertaufgabe für die hydrodynamischen Grundgleichungen // Math. Nachr. 1951. V. 4. P. 213-231.

[25] T. Kato. Abstract evolution equations of parabolic type in Banach and Hilbert spaces // Nagoya Math. J. 1961. V. 19. P. 93-125.

[26] О. А. Ладыженская. Математические вопросы динамики вызкой несжимаемой жидкости. М.: Наука, 1970.

[27] О. А. Ладыженская, В. А. Солонников, Н.Н. Уральцева. Линейные и квазилинейные уравнения параболического типа. М.: Мир, 1985.

[28] J.-B. Leblond. Mathematical results for a model of diffusion and precipitation of chemical elements in solid matrices // Nonlinear Anal. Real World Appl. 2005. V. 6. № 2. P. 297-322.

[29] G. M. Lieberman. Second Order Parabolic Differential Equations. River Edge, NJ: World Scientific, 1996.

[30] J.-L. Lions. Équations différentielles opérationelles et problèmes aux limites. Berlin: Springer-Verlag, 1961.

[31] A. Lunardi. Analytic Semigroups and Optimal Regularity in Parabolic Problems. Basel: Birkhäuser, 1995. (Progr. Nonlinear Differential Equations Appl. V. 16.)

[32] J. Meixner. On the linear theory of heat conduction // Arch. Ration. Mech. Anal. 1971. V. 39. P. $108-130$

[33] M. Nitzberg, T. Shiota. Nonlinear image filtering with edge and corner enhancement // IEEE Trans. Pattern Anal. Machine Intelligence. 1992. V. 14. № 8. P. 826-833.

[34] J. W. Nunziato. On heat conduction in materials with memory // Quart. Appl. Math. 1971. V. 29. P. 187-204.

[35] P. Perona, J. Malik. Scale-space and edge detection using anisotropic diffusion // IEEE Trans. Pattern Anal. Machine Intelligence. 1990. V. 12. № 7. P. 629-639.

[36] J. Prüss. Maximal regularity for evolution equations in $L_{p}$-spaces // Conf. Semin. Mat. Univ. Bari. 2002 (2003). № 285. P. 1-39.

[37] G. Simonett. Center manifolds for quasilinear reaction-diffusion systems // Differential Integral Equations 1995. V. 8. № 4. P. 753-796.

[38] G. Simonett. Invariant manifolds and bifurcation for quasilinear reaction-diffusion systems // Nonlinear Anal. 1994. V. 23. № 4. P. 515-544.

[39] П. Е. Соболевский. Уравнения параболического типа в банаховомпространстве // Труды ММО. 1961. Т. 10. С. 297-350.

[40] М. И. Вишик, О. А. Ладыженская. Краевые задачи для уравнений в частных производных и некоторых классов операторных уравнений // УМН. 1956. Т. 11. № 6. С. 41-97.

[41] L. Weis. Operator-valued Fourier multiplier theorems and maximal $L_{p}$-regularity // Math. Ann. 2001. V. 319. № 4. P. 735-758.

[42] S. Zheng. Nonlinear Evolution Equations. Boca Raton, FL: Chapman \& Hall/CRC, 2004. (Chapman \& Hall/CRC Monogr. Surv. Pure Appl. Math., V. 133.)

Institut für Mathematik, Universität Zürich, Switzerland

Поступила в редакцию 